J. Clin. Chem. Clin. Biochem.

Vol. 20, 1982, pp. 235-242

\title{
Gemeinsame Studie zur Erstellung von Richtwerten für klinisch-chemische Kenngrößen im Kindesalter
}

Von I. Witt

Klinisch-Chemisches und Biochemisches Laboratorium der Kinderklinik der Universität Freiburg i. Br. und

\author{
Chr. Trendelenburg
}

Klinisch-Chemisches Laboratorium der Chirurgischen Klinik der Universität Freiburg i. Br.

\section{Beteiligte:}

F. Bidlingmaier, Universitäts-Kin derklinik, München

G. Brügmann, Universitäts-Kinderklinik, Tübingen

$K$. Dörner, Universitäts-Kinderklinik, Kiel

H. J. Gibitz, Landeskrankenanstalten, Salzburg

H. Helwig, Kinderkrankenhaus St. Hedwig, Freiburg

G. Klein, Kinderkrankenhaus Rothenburgsort, Hamburg

I. Kupke, Universitäts-Kinderklinik, Düsseldorf

(Eingegangen am 1. Juni 1981/4. Januar 1982)
N. Liappis, Universitäts-Kinderklinik, Bonn

D. Mathias, Universitäts-Kinderklinik, Heidelberg

$H$. Schall, Universitäts-Kinderklinik, Frankfurt a/M

R. D. Schmid, Klinikum, Nürnberg

F. C. Sitzmann், Universitäts-Kinderklinik, Homburg/Saar

I. Witt, Universitäts-Kinderklinik, Freiburg

U. Wulff, Kinderklinik der Med. Hochschule Lübeck

Zusammenfassung: Der Mangel an zuverlässigen Bezugswerten für klinisch-chemische Kenngrößen im Kindesalter veranlaßte die Arbeitsgruppe ,Pädiatrische Klinische Chemie‘, eine gemeinsame Studie zur Erstellung von Referenzwerten durchzuführen. In 15 Kliniken wurden Werte für die wichtigsten klinisch-chemischen Kenngrößen bei Kindern verschiedener Altersgiuppen ermittelt. Die in die Studie einbezogenen Kinder erfüllten die hinsichtlich ihres Gesundheitszustandes geforderten Minimalkriterien.

Die Auswertung der Daten erfolgte aufgeteilt nach Kenngröße und Altersgruppe. És werden die Percentile sowie die Mittelwerte und 95\%-Vertrauensbereiche angegeben. Aus den ermittelten Referenzwerten wurden Richtwerte abgeleitet, die für die tägliche Routinearbeit praktikabel sind.

\section{Joint study to establish reference values for clinical chemical parameters in childhood}

Summary: The working group 'Pädiatrische Klinische Chemie' undertook a joint study to satisfy the need for more reliable reference values for clinical chemical parameters in childhood.

In 15 different pediatric hospitals data for the most important clinical chemical parameters were determined for children of different age groups. The children included in the study fulfilled the required minimal criteria concerning their health state.

The data were listed according to parameter and age group and the percentiles and mean values with $95 \%$ confidence intervals are presented. From these values, reference ranges are derived that are suitable for daily routine analyses.

\section{Einftihrung}

Bei der Beurteilung von klinisch-chemischen Kenngrößen im Kindesalter stoßen sowohl Laborleiter als auch Ärzte immer wieder auf das Problem, welche ,Normalwerte“ dazu herangezogen werden können. Eine Umfrage bei zahlreichen Universitäts-Kinderkliniken in Deutschland, die 1976 durchgeführt wurde, ergab, daß sich die in den jeweiligen Kliniken verwendeten ,Normalwerte‘ auch bei Verwendung gleicher Methoden erheblich voneinander unterscheiden. Meistens sind die Bezugswerte aus der Literatur übernommen. Nur in wenigen Fällen sind sie in den Kliniklaboratorien selbst ermittelt worden. Das ist verständlich, denn es ist fast unmöglich, für alle wichtigen Kenngrößen und alle Altersstufen genügend 
Blutproben von gesunden Kindern zu bekommen. Die in der Literatur mitgeteilten Werte sind aber nicht immer mit den im jeweiligen Labor verwendeten Methoden ermittelt worden und zeigen häufig eine unbrauchbare Einteilung in die verschiedenen Altersklassen. Außerdem findet man für viele Kenngrößen stark voneinander abweichende Angaben, und einige der in der Literatur angegebenen Werte stimmen nicht mit den Erfahrungswerten überein (vgl. 1.c. $(20,21,22))$.

Um diese seit Jahren bekannte mißliche Situation zu verbessern, beschloß die 1976 ins Leben gerufene Arbeitsgruppe ,Pädiatrische Klinische Chemie', eine gemeinsame Studie zur Erstellung von zuverlässigen Bezugswerten durchzufuihren. Bei der Planung der Studie wurde beschlossen, die Bezeichnung ,Normalwert' aufzugeben und künftig nur noch von ,Referenzwert ${ }^{\text {‘ }}$ und ,Richtwert' zu sprechen.

Der Begriff ,Richtwert' wurde definiert als, obere bzw. untere Grenze des Warnbereichs'. Diese Definition impliziert, daß der Begriff Richtwert bzw. Richtwertbereich weiter gefaßt ist als der sonst gebräuchliche Begriff des Normalwertes. Einmal ist heute nicht genau zu definieren, was ,normal' bedeutet, zum anderen sollen die Richtwerte von der ,Zahlengläubigkeit' fortführen und eine sinnvolle Einordnung der klinischchemischen Kenngrößen in das Gesamtbild des Patienten bewirken.

Die den Richtlinien zugrunde liegenden Meßwerte sollen als Referenzwerte bezeichnet werden, da sie an einem definierten Kollektiv erhoben wurden.

Die gemeinsame Studie wurde entsprechend dem 1976 festgelegten Plan durchgeführt. Obwohl zahlreiche Hindernisse zu überwinden waren, liegt jetzt ein brauchbares Ergebnis der recht mühevollen Arbeit vor, über das im folgenden berichtet wird.

\section{Durchfuihrung der Studie}

In die Studie wurden nur die wichtigsten klinisch-chemischen Kenngrößen einbezogen, bei denen nicht unbedingt definierte Bedingungen bei der Probennahme (z. B. am nüchternen Probanden) eingehalten werden mußten. Zur Bestimmung der Kenngrößen wurden jeweils verschiedene der heute gebräuchlichen Methoden verwendet. In Tabelle 1 sind die untersuchten Kenngrößen und die verwendeten Methoden zusammengestellt.

Jeder Teilnehmer ermittelte für eine Anzähl der festgelegten Kenngrößen pro Altersgruppe (Tab. 2) zwischen 5 und 20 Werte. Die Dokumentation der Werte erfolgte zusammen mit der Angabe der verwendeten Methode und der durchgeführten internen Qualitätskontrolle. Alle beteiligten Laboratorien erfullten außerdem die Anforderungen der gesetzlich vorgeschriebenen externen Qualitätskontrolle.

Während der zweiten Zeitspanne der Studie wurden von den meisten der beteiligten Laboratorien zusätzlich zu der oben erwähnten Qualitätskontrolle alle Kenngrößen im gleichen Kontrollserum analysiert. Die Daten dieses, Ringversuches wurden nur verwendet zur Beurteilung, ob erhebliche methodische oder labortechnische Abweichungen vorlagen.
Tab. 1. Zusammenstellung der Kenngrößen, fứr die Richtwerte ermittelt wurden, sowie Angabe der verwendeten Methoden.

\begin{tabular}{|c|c|}
\hline Kenngröße & Methoden \\
\hline Harnstoff & $\begin{array}{l}\text { Urease-Spaltung (Berthelot-Reaktion) (1) } \\
\text { BUN }^{1} \text { )-Analyzer (Beckman) (2) }\end{array}$ \\
\hline Kreatinin & $\begin{array}{l}\text { Jaffé-Reaktion nach Enteiweißung mit } \\
\text { Tríichloressigsäure (3) } \\
\tilde{J}_{a f f e ́} \text {-Reaktion ohne Enteiw̄eißung (4) } \\
\text { Kinetisch: Creatinin-Analyzer (Beckman) } \\
\text { (5) }\end{array}$ \\
\hline Harnsäure & $\begin{array}{l}\text { Uricase-Spaltung (6) } \\
\text { Kageyama-Reaktión (7) }\end{array}$ \\
\hline Gesamt-Eiweiß & $\begin{array}{l}\text { Biuret-Methode mit und ohne Berücksich: } \\
\text { tigung des Serumleèrwêrtes (8) }\end{array}$ \\
\hline Natrium & Flàmmenphotometrie \\
\hline Kạlium & Flummenphotometrie \\
\hline Calcium & $\begin{array}{l}\text { Flammenphotometrie } \\
\text { Atomabsorption } \\
\text { Kömplexometrische Titration }\end{array}$ \\
\hline Chlorid & Coulometrische Titration \\
\hline Anorgan. Phosphor & $\begin{array}{l}\text { Reaktion mit Ammoniummolybdat, Redu } \\
\text { zu Molybdänblau } \\
\text { (mit uñd ohne Enteiweißung) (9) }\end{array}$ \\
\hline Eisen & $\begin{array}{l}\text { Bathophenanthrolin } \\
\text { mit und ohne Enteiweißung }(10,11) \\
\text { Ferrozin }(12)\end{array}$ \\
\hline Bilirubin & $\begin{array}{l}\text { Photometrie des Ażopigments } \\
\text { (Jendrassik/Grof) (13) } \\
\text { Dichlorphenyldiażonium-Methòde (14) }\end{array}$ \\
\hline Cholesterin & $\begin{array}{l}\text { Enzymatischer Farbtest mit Cholesterin- } \\
\text { esterase/oxidase und Katalase (15) bzw. } \\
\text { Phenol/4-Aminophenazon (16) }\end{array}$ \\
\hline $\begin{array}{l}\text { Glutamat-Oxalacetat } \\
\text { Transaminase }\end{array}$ & $\begin{array}{l}\text { Standardmethode der Dt. Ges. für } \\
\text { Klinische Chemie } \\
\text { (optimiert) (17) }\end{array}$ \\
\hline $\begin{array}{l}\text { Glutamat-Pyruvat- } \\
\text { Transaminase }\end{array}$ & $\begin{array}{l}\text { Standardmethode der Dt. Ges: für } \\
\text { Klinische Chemie } \\
\text { (optimiert) (17) }\end{array}$ \\
\hline $\begin{array}{l}\boldsymbol{\gamma} \text {-Glutamyl- } \\
\text { Transpeptidase }\end{array}$ & Mẹthode nach Szász (18) \\
\hline $\begin{array}{l}\text { Alkalische } \\
\text { Phosphatase }\end{array}$ & $\begin{array}{l}\text { Standardmethode der Dt. Ges. für } \\
\text { Klinische Chemie } \\
\text { (optimiert) (17) }\end{array}$ \\
\hline $\begin{array}{l}\text { Lactat- } \\
\text { Dehydrogenase }\end{array}$ & $\begin{array}{l}\text { Standardmethode der Dt. Ges. für } \\
\text { Klinische Chemie } \\
\text { (optimiert) (17) }\end{array}$ \\
\hline Kreatinkinase & $\begin{array}{l}\text { Standardmethode der Dt. Ges. für } \\
\text { Klinische Chemie } \\
\text { Aktivierung durch N-Acetyl-cystein (19) }\end{array}$ \\
\hline
\end{tabular}

1) Harnstoff-N im Blut

Tab. 2. Verwendete Altersstufen-Einteilung

$I=$ Neugeborene 1. Tag-4. Woche

II $\equiv$ Säuglinge 2.-12. Monat

III = Kinder nach 12. Monat

\section{Ausnahmen}

Natrium

I = Neugeborene

II = Säuglinge 1 Halbjạhr

III = Säuglinge und Kinder $a b 6$. Monat

Alkalische Phosphatase

$\mathrm{I}=1 .-10$. Tag

II $=10 .-30$. Tàg

III $=2 .-12$. Monat

$\mathrm{IV} \doteq 2 .-8 . \mathrm{Jahr}$

$\mathrm{V}=\mathrm{ab} 8 \mathrm{Jahre}$ 
Die in die Studie aufgenommenen Kinder mußten festgelegte Minimalkriterien erfillen, die in Tabelle 3 dargestellt sind. Es handelte sich dabei entweder um Kinder von Klinikangehörigen oder um Kinder, die zu präoperativen Kontrolluntersuchungen in die Klinik kamen und bei denen eine venöse Blutentnahme indiziert war.

Die Daten wurden nach Kenngröße und Altersstufe aufgeteilt, als Histogramme dargestellt und hinsichtlich der Streuungsmaße (Percentilwerte, Mittelwerte und 95\%-Vertrauensbereiche) beschrieben.

Tab. 3. Minimalkriterien zur Einbeziehung von,gesunden' Kindern in die gemeinsame Studie zur Erstellung von Richtwerten.

Folgende Voraussetzungen müssen erfullt sein:

1. Normale Entwicklung.

2. Keine chronischen Erkrankungen.

3. z. Zt. keine akuten Erkrankungen.

4. z. Zt. keinerlei Therapie oder Medikamenteneinnahme.

5. Bei körperlicher Untersuchung kein pathologischer Befund (Ausnahmen: Leistenhernie, Maldescensus).

6. Kein pathologischer Befund bei histologischer oder röntgenologischer Untersuchung.

7. Bei Neugeborenen keine akute oder chronische Erkrankung der Mutter.

8. Bei Neugeborenen darf die Bilirubinkonzentration nicht über $240 \mu \mathrm{mol} / 1$ liegen.

\section{Ergebnis der Studie}

Beim Vergleich der Werte, die mit verschiedenen Methoden ermittelt wurden, zeigte sich, daß die biologische Varianz innerhalb der gleichen Altersstufe wesentlich größer ist als methodisch bedingte Unterschiede. Daher wurden die in Tabelle 1 für eine Kenngröße angegebenen Methoden zusammen ausgewertet.

Von den vorliegenden Werten wurden die Mittelwerte und die 95\%-Vertrauensbereiche (Tab. 4) sowie die Percentile (Tab. 5) berechnet. Für viele Kenngrößen ist der Verteilungstyp unbekannt und die Anzahl der in unserer Studie erhobenen Werte häufig zu gering, um den Verteilungstyp zu bestimmen. Deswegen wurden bei der Festlegung vón Richtwerten sowôhl

Tab. 4. Referenzwerte (Mittelwerte und 95\%:Vertrauensbereich).

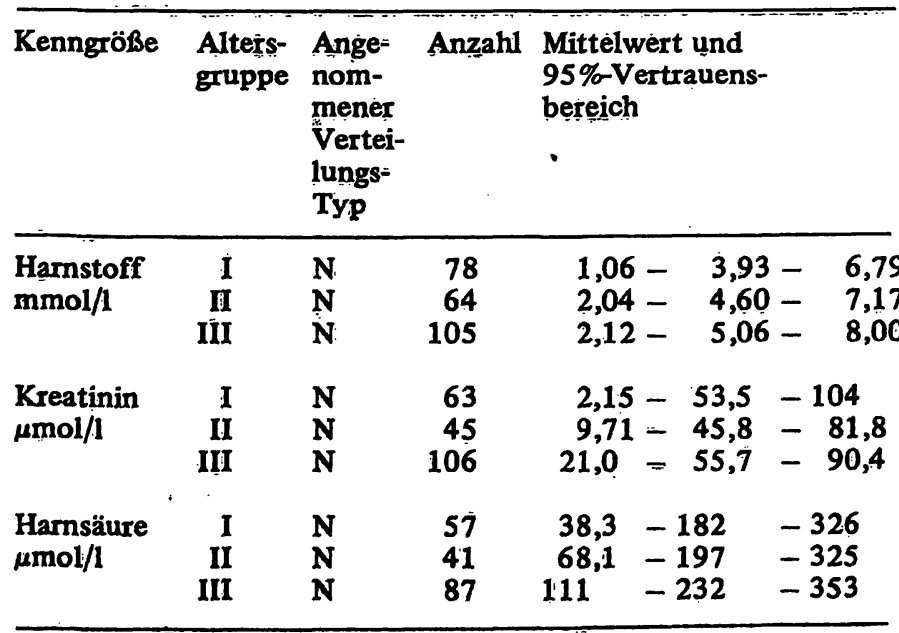

Tab. 4. Fortsetzung

\begin{tabular}{|c|c|c|c|c|c|c|}
\hline Kenngröße & $\begin{array}{l}\text { Alters- } \\
\text { gruppe }\end{array}$ & $\begin{array}{l}\text { Ange- } \\
\text { nom- } \\
\text { mener }\end{array}$ & Anzahl & $\begin{array}{l}\text { Mittelw } \\
95 \%-\mathrm{Ve} \\
\text { bereich }\end{array}$ & $\begin{array}{l}\text { vert und } \\
\text { ertrauens- }\end{array}$ & \\
\hline $\begin{array}{l}\text { Eiweiß } \\
\text { g/1 }\end{array}$ & III & $\begin{array}{l}\mathbf{N} \\
\mathbf{N} \\
\mathbf{N}\end{array}$ & $\begin{array}{r}83 \\
49 \\
101\end{array}$ & $\begin{array}{l}45,2 \\
45,7 \\
58,5\end{array}$ & $\begin{array}{l}56,9 \\
-\quad 59,5 \\
-\quad 69,3\end{array}$ & $\begin{array}{l}-68,6 \\
-\quad 73,3 \\
-\quad 80,1\end{array}$ \\
\hline $\begin{array}{l}\text { Natrium } \\
\mathrm{mmol} / 1\end{array}$ & $\underset{\text { III }}{\text { II }}$ & $\begin{array}{l}\mathbf{N} \\
\mathbf{N} \\
\mathbf{N}\end{array}$ & $\begin{array}{r}78 \\
47 \\
122\end{array}$ & $\begin{array}{l}132 \\
129 \\
132\end{array}$ & $\begin{array}{l}-140 \\
-136 \\
-139\end{array}$ & $\begin{array}{l}-147 \\
-143 \\
-145\end{array}$ \\
\hline $\begin{array}{l}\text { Kalium } \\
\text { mmol/1 }\end{array}$ & II & $\begin{array}{l}\mathbf{N} \\
\mathbf{N} \\
\mathbf{N}\end{array}$ & $\begin{array}{r}78 \\
64 \\
105\end{array}$ & $\begin{array}{l}3,56 \\
3,65 \\
3,13\end{array}$ & $\begin{array}{r}5,84 \\
-\quad 4,74 \\
-\quad 4,14\end{array}$ & $\begin{array}{l}4-6,11 \\
4-\quad 5,83 \\
4-\quad 5,15\end{array}$ \\
\hline $\begin{array}{l}\text { Calcium } \\
\mathrm{mmol} / 1\end{array}$ & $\underset{\text { III }}{\text { I }}$ & $\begin{array}{l}\mathbf{N} \\
\mathbf{N} \\
\mathbf{N}\end{array}$ & $\begin{array}{r}87 \\
63 \\
107\end{array}$ & $\begin{array}{l}1,76 \\
2,04 \\
2,09\end{array}$ & $\begin{array}{l}2,27 \\
-\quad 2,39 \\
-\quad 2,35\end{array}$ & $\begin{array}{l}7-\quad 2,78 \\
9-\quad 2,73 \\
5-\quad 2,61\end{array}$ \\
\hline $\begin{array}{l}\text { Chlorid } \\
\mathrm{mmol} / \mathrm{l}\end{array}$ & $\underset{\text { III }}{\text { I }}$ & $\begin{array}{l}\mathbf{N} \\
\mathbf{N} \\
\mathbf{N}\end{array}$ & $\begin{array}{l}85 \\
52 \\
83\end{array}$ & $\begin{array}{l}95,2 \\
92,9 \\
94,5\end{array}$ & $\begin{array}{l}-105 \\
-103 \\
-103\end{array}$ & $\begin{array}{l}-116 \\
-112 \\
-111\end{array}$ \\
\hline $\begin{array}{l}\text { Anorgan. } \\
\text { Phosphor } \\
\text { mmol/l }\end{array}$ & III & $\begin{array}{l}\mathbf{N} \\
\mathbf{N} \\
\mathbf{N}\end{array}$ & $\begin{array}{l}81 \\
51 \\
93\end{array}$ & $\begin{array}{l}1,56 \\
1,58 \\
1,09\end{array}$ & $\begin{array}{r}2,32 \\
-\quad 2,06 \\
-\quad 1,54\end{array}$ & $\begin{array}{l}2-3,08 \\
6-\quad 2,54 \\
4-\quad 2,00\end{array}$ \\
\hline $\begin{array}{l}\text { Eisen } \\
\mu \mathrm{mol} / 1\end{array}$ & III & $\begin{array}{l}\text { L } \\
\text { L } \\
\text { L }\end{array}$ & $\begin{array}{l}69 \\
50 \\
97\end{array}$ & $\begin{array}{l}6,41 \\
6,44 \\
7,68\end{array}$ & $\begin{array}{l}14,5 \\
-\quad 13,5 \\
-\quad 16,0\end{array}$ & $\begin{array}{l}5-32,7 \\
5-28,2 \\
0-33,4\end{array}$ \\
\hline $\begin{array}{l}\text { Bilirubin } \\
\mu \mathrm{mol} / 1\end{array}$ & III & $\begin{array}{l}\mathbf{L} \\
\mathbf{L}\end{array}$ & $\begin{array}{l}39 \\
80\end{array}$ & $\begin{array}{l}1,73 \\
1,70\end{array}$ & $\begin{array}{l}4,89 \\
-\quad 6,05\end{array}$ & $\begin{array}{l}9-13,8 \\
5-21,5\end{array}$ \\
\hline $\begin{array}{l}\text { Cholesterin } \\
\text { mmol/1 }\end{array}$ & III & $\begin{array}{l}\mathbf{N} \\
\mathbf{N} \\
\mathbf{N}\end{array}$ & $\begin{array}{l}77 \\
58 \\
86\end{array}$ & $\begin{array}{l}1,37 \\
1,51 \\
2,89\end{array}$ & $\begin{array}{r}2,93 \\
-\quad 3,24 \\
-\quad 4,35\end{array}$ & $\begin{array}{l}3-\quad 4,49 \\
4-\quad 4,97 \\
5-\quad 5,81\end{array}$ \\
\hline $\begin{array}{l}\text { Glutamat- } \\
\text { Oxalacetat- } \\
\text { Trans- } \\
\text { aminase } \\
(\mathrm{U} / 1)\end{array}$ & III & $\begin{array}{l}\mathbf{N} \\
\mathbf{N} \\
\mathbf{N}\end{array}$ & $\begin{array}{r}85 \\
63 \\
106\end{array}$ & $\begin{array}{l}5,91 \\
7,38 \\
4,70\end{array}$ & $\begin{array}{l}-21,9 \\
-\quad 17,3 \\
-\quad 13,4\end{array}$ & $\begin{array}{l}-37,9 \\
-\quad 27,3 \\
-\quad 22,2\end{array}$ \\
\hline $\begin{array}{l}\text { Glutamat- } \\
\text { Pyruvat- } \\
\text { Trans- } \\
\text { aminase } \\
\text { U/1 }\end{array}$ & IIII & $\begin{array}{l}\mathbf{L} \\
\mathbf{L} \\
\mathbf{L}\end{array}$ & $\begin{array}{r}73 \\
53 \\
106\end{array}$ & $\begin{array}{l}4,47 \\
6,23 \\
4,50\end{array}$ & $\begin{array}{l}-12,4 \\
-\quad 14,9 \\
-\quad 9,61\end{array}$ & $\begin{array}{l}-32,4 \\
-\quad 35,7 \\
-\quad 20,5\end{array}$ \\
\hline $\begin{array}{l}\text { r-Glutamyl- } \\
\text { Trans- } \\
\text { peptidase } \\
\text { U/1 }\end{array}$ & IIII & $\begin{array}{l}\mathrm{L} \\
\mathrm{L} \\
\mathrm{L}\end{array}$ & $\begin{array}{l}69 \\
44 \\
97\end{array}$ & $\begin{array}{r}13,9 \\
1,95 \\
3,10\end{array}$ & $\begin{array}{l}-47,8 \\
-\quad 13,3 \\
-\quad 7,32\end{array}$ & $\begin{array}{l}-163 \\
-90,8 \\
-\quad 17,3\end{array}$ \\
\hline $\begin{array}{l}\text { Kreatinkinase } \\
\text { U/1 }\end{array}$ & III & $\begin{array}{l}\mathbf{L} \\
\mathbf{L}\end{array}$ & $\begin{array}{l}29 \\
66\end{array}$ & $\begin{array}{l}16,7 \\
15,6\end{array}$ & $\begin{array}{l}-47,7 \\
-\quad 38,3\end{array}$ & $\begin{array}{l}-136 \\
-\quad 93,8\end{array}$ \\
\hline $\begin{array}{l}\text { Lactat- } \\
\text { Dehydro- } \\
\text { genase } \\
\text { U/1 }\end{array}$ & $\underset{\text { III II }}{\text { İ }}$ & $\begin{array}{l}\text { L } \\
\mathbf{L} \\
\mathbf{L}\end{array}$ & $\begin{array}{l}56 \\
51 \\
70\end{array}$ & $\begin{array}{l}200 \\
156 \\
131\end{array}$ & $\begin{array}{l}-410 \\
-285 \\
-213\end{array}$ & $\begin{array}{l}-838 \\
-521 \\
-344\end{array}$ \\
\hline $\begin{array}{l}\text { Alkalische } \\
\text { Phosphatase } \\
\text { U/1 }\end{array}$ & $\begin{array}{l}\text { I } \\
\text { III } \\
\text { IV } \\
\text { V }\end{array}$ & $\begin{array}{l}\mathbf{N} \\
\mathbf{N} \\
\mathbf{N} \\
\mathbf{N} \\
\mathbf{N}\end{array}$ & $\begin{array}{l}71 \\
42 \\
66 \\
91 \\
90\end{array}$ & $\begin{array}{l}116 \\
135 \\
159 \\
128 \\
114\end{array}$ & $\begin{array}{l}-240 \\
-363 \\
-421 \\
-360 \\
-343\end{array}$ & $\begin{array}{l}-364 \\
-591 \\
-683 \\
-593 \\
-572\end{array}$ \\
\hline
\end{tabular}

$\mathrm{N}=$ Normalverteilung

$L=\log$. Normalverteilung 
Tab. 5. Referenzwerte (Percentile).

\begin{tabular}{|c|c|c|c|c|c|c|c|c|}
\hline Percentile & & 0,025 & 0,050 & 0,100 & 0,500 & 0,900 & 0,950 & 0,975 \\
\hline \multicolumn{9}{|l|}{$\begin{array}{l}\text { Harnstoff (mmol/1) } \\
\quad(\mathrm{N}=78)\end{array}$} \\
\hline $\begin{aligned} \text { I } & (\mathrm{N}=78) \\
\text { II } & (\mathrm{N}=64) \\
\text { III } & (\mathrm{N}=105)\end{aligned}$ & $\begin{array}{l}0,68 \\
1,35 \\
1,67\end{array}$ & $\begin{array}{l}1,14 \\
2,40 \\
2,82\end{array}$ & $\begin{array}{l}1,40 \\
2,59 \\
2,96\end{array}$ & $\begin{array}{l}2,21 \\
2,84 \\
3,26\end{array}$ & $\begin{array}{l}3,93 \\
4,63 \\
4,96\end{array}$ & $\begin{array}{l}5,62 \\
6,06 \\
7,04\end{array}$ & $\begin{array}{l}6,12 \\
6,74 \\
7,77\end{array}$ & $\begin{array}{l}6,83 \\
7 ; 11 \\
8,46\end{array}$ \\
\hline
\end{tabular}

Kreatinin $(\mu \mathrm{mol} / \mathrm{l})$

I $\quad(\mathrm{N}=63)$

II $\quad\left(\begin{array}{rl}N \\ \text { III } \quad(N)\end{array}\right.$

$\begin{array}{cc}8,84 & 12,4 \\ 15,9 & 17,7\end{array}$

$13,3 \quad 26,1$

4,96

7,04

6,74
7,77

8,97

Harnsäure ( $\mu \mathrm{mol} / \mathrm{l})$

$\begin{array}{ll}\text { I } & (\mathrm{N}=57) \\ \text { II } & (\mathrm{N}=41) \\ \text { III } & (\mathrm{N}=87) \\ \text { Gesamt-Eiweiß (g/l) } \\ \text { I } \quad(\mathrm{N}=83)\end{array}$

II $\quad(\mathrm{N}=49)$

13,3

24,3

$19,4 \quad 20,8$

50,4

89,3

102

$110 \quad 120$

$30,9 \quad 35,4$

53,0

83,5

84,0
88,4

88,4
88,4

88,4

106

III $\quad(\mathrm{N}=101)$

$\begin{array}{cc}83,3 & 89,2 \\ 113 & 114 \\ 110 & 120\end{array}$

$\begin{array}{ll}101 & 113 \\ 117 & 119\end{array}$

161
196

196
226

306
277

277

335

328

353

371

372

381

$149 \quad 160$

300

362

422

Natrium (mmol/1) :

I $\quad(\mathrm{N}=78)$

II $\quad(N=47)$

$\begin{array}{lll}42,0 & 46,1 & 48,5 \\ 48,0 & 48,0 & 48,5\end{array}$

50,0

56,0

$\begin{array}{llll}65,0 & 66,5 & 67,5 & 78,0\end{array}$

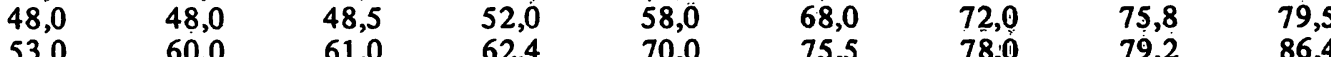

Kalium (mmol/1)

I $\quad(\mathrm{N}=78)$

II $\quad(\mathrm{N}=64)$

1

$60,0 \quad 61,0$

$62,470,0$

75,5

78,0

79,2

86,4

III $\quad(\mathrm{N}=105)$
Calcium $(\mathrm{mmol} / \mathrm{l})$

$\begin{aligned} \text { I } & (\mathrm{N}=87) \\ \text { II } & (\mathrm{N}=63) \\ \text { III } & (\mathrm{N}=107)\end{aligned}$

$130 \quad 130$

$128 \quad 129$

133
130

136
132

140
137
138

144
140
142

146
141

147
142

151

$\begin{array}{lll}143 & 14 \hat{6} & 142 \\ & & 148\end{array}$

$\begin{array}{ll}3,10 & 3,63 \\ 2,90 & 3,68 \\ 3,00 & 3,20\end{array}$

\section{3,90}

4,10

3,93
3,60

\section{4,80}

4,78

4,10

5,80

5,35

4,80

5,85

5,55

6,03

5,70

6,20

5,15

5,35

5,95

6,00

Chlorid (mmol/1)

$\begin{aligned} \text { II } & (\mathrm{N}=85) \\ \text { II } & (\mathrm{N}=52)\end{aligned}$

$1,49 \quad 1,52$

$1,74 \quad 1,95$

2,30

2,54

2,63

2,68

2,77

$\begin{array}{llll}1,70 & 2,10 & 2,15 & 2,20 \\ 1,95 & 2,06 & 2,13 & 2,20\end{array}$

2,40
2,35

2,64

2,68

2,70

2,75

2,53

$$
2,5 \overline{5}
$$

2,57

2,62

III $(\mathrm{N}=83)$

$\begin{array}{ll}95,0 & 96,0 \\ 88,0 & 89,5\end{array}$

\section{6,5}

\section{8,5}

105

$$
111
$$

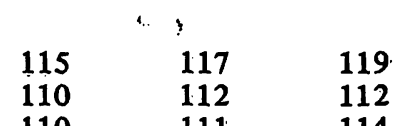

$\begin{array}{lllllll}96,0 & 97,0 & 102 . & 108 & 110 & 111 & 114\end{array}$

Phosphor (mmol/l)

$$
\text { II } \quad(\mathrm{N}=81)
$$$$
94,0 \quad 95,5
$$

$96,0 \quad 97,0 \quad 102 . \quad 108$

$\begin{array}{ll}1,29 & 1,45 \\ 1,52 & 1,55 \\ 0,94 & 1,04\end{array}$

1,63
1,73
1,18

2,00

2,00
1,80

2,29

2,03
1,55

2,74
2,38
1,86

2,88
2,44

3,46

2,58

3,57

Eisen $(\mu \mathrm{mol} / \mathrm{l})$

$\begin{aligned} \text { I } & (\mathrm{N}=69) \\ \text { II } & (\mathrm{N}=50) \\ \text { III } & (\mathrm{N}=97)\end{aligned}$

\section{2,33}

\section{6,00}

\section{6,81}

8,60

15,8

22,9

\section{5,8}

2,05

2,60

$\begin{array}{llll}4,12 & 5,46 & 6,99 & 8,06\end{array}$

15,8
13,9

22,9

$25,8 \quad 26 ; 3$

$26 ; 3 \quad 27,2$

$8,15 \quad 9,85$

$16,8 \quad 24,0$

$\begin{array}{ll}21,9 & 25,9 \\ 27,9 & 30,7\end{array}$

26,9

Bilirubin ( $\mu \mathrm{mol} / \mathrm{l})$

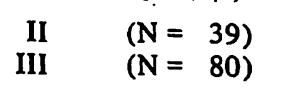

$1,88 \quad 1,97$

$2,22 \cdot 3,08$

4,79

9,66

\begin{tabular}{|c|c|c|c|c|c|c|c|c|c|c|}
\hline $\begin{array}{r}\text { II } \\
\text { III }\end{array}$ & $\begin{array}{l}(\mathrm{N}=77) \\
(\mathrm{N}=58) \\
(\mathrm{N}=86)\end{array}$ & $\begin{array}{l}1,32 \\
1,68 \\
1,68\end{array}$ & $\begin{array}{l}1,39 \\
1,79 \\
2,68\end{array}$ & $\begin{array}{l}1,72 \\
2,06 \\
2,85\end{array}$ & $\begin{array}{l}2,11 \\
2,20 \\
3,45\end{array}$ & $\begin{array}{l}2,77 \\
3,11 \\
4,43\end{array}$ & $\begin{array}{l}4,03 \\
4,49 \\
5,15\end{array}$ & $\begin{array}{l}4,18 \\
4,82 \\
5,43\end{array}$ & $\begin{array}{l}4,49 \\
5,21 \\
5,50\end{array}$ & $\begin{array}{r}5,49 \\
.5,47 \\
5,91\end{array}$ \\
\hline \multicolumn{11}{|c|}{ Glutamat-Oxalacetat-Transaminase (U/1) } \\
\hline $\begin{array}{r}\text { I } \\
\text { II } \\
\text { III }\end{array}$ & $\begin{array}{l}(N=85) \\
(N=63) \\
(N=106)\end{array}$ & $\begin{array}{l}7,70 \\
4,30 \\
5,70\end{array}$ & $\begin{array}{l}8,90 \\
6,55 \\
6,00\end{array}$ & $\begin{array}{l}9,15 \\
9,50 \\
6,60\end{array}$ & $\begin{array}{c}13,0 \\
12,0 \\
9,00\end{array}$ & $\begin{array}{r}20,0 \\
17,0 \\
\cdot 14,0\end{array}$ & $\begin{array}{l}34,5 \\
24 ; 0 \\
17,0\end{array}$ & $\begin{array}{l}38,0 \\
26,5 \\
18 ; 6\end{array}$ & $\begin{array}{c}39 ; 0 \\
27,5 \\
22,1 \\
\vdots\end{array}$ & $\begin{array}{l}43,0 \\
31,0 \\
39,0\end{array}$ \\
\hline
\end{tabular}

$30,7 \quad 33,7$

Cholesterin (mmol/l) 
Tab. 5. Fortsetzung

\begin{tabular}{llllllll}
\hline Percentile & 0,025 & 0,050 & 0,100 & 0,500 & 0,900 & 0,950 & 0,975
\end{tabular}

Glutamat-Pyruvat-Transaminase (U/1)

\begin{tabular}{|c|c|c|c|c|c|c|c|c|c|}
\hline II & $\begin{array}{l}(N=73) \\
(N=53) \\
(N=106)\end{array}$ & $\begin{array}{l}3,00 \\
5,90 \\
4,00\end{array}$ & $\begin{array}{l}5,00 \\
6,45 \\
5,00\end{array}$ & $\begin{array}{l}5,70 \\
7,10 \\
5,10\end{array}$ & $\begin{array}{l}6,20 \\
8,00 \\
6,55\end{array}$ & $\begin{array}{c}13,0 \\
16,0 \\
9,00\end{array}$ & $\begin{array}{l}21,5 \\
25,0 \\
16,5\end{array}$ & $\begin{array}{l}23,6 \\
27,5 \\
20,3\end{array}$ & $\begin{array}{l}29,5 \\
31,0 \\
24,1\end{array}$ \\
\hline
\end{tabular}

$\gamma$-Glutamyl-Transpeptidase (U/1)

$\begin{array}{rll}\text { I } & (\mathbf{N}=69) & 8,00 \\ \text { II } & (\mathbf{N}=44) & 3,00 \\ \text { III } & (\mathbf{N}=97) & 3,00\end{array}$

$\begin{array}{cccccccc}13,0 & 15,5 & 19,5 & 48,0 & 95,3 & 130 & 146 & 162 \\ 3,50 & 4,00 & 4,50 & 11,8 & 63,8 & 99,9 & 177 & 222 \\ 3,20 & 4,00 & 4,00 & 7,00 & 12,1 & 14,1 & 22,4 & 28,9\end{array}$

Lactat-Dehydrogenase (U/1)

$\begin{array}{rrrr}\text { I } & (\mathrm{N}=56) & 136 & 158 \\ \text { II } & (\mathrm{N}=51) & 144 & 153 \\ \text { III } & (\mathrm{N}=70) & 74,8 & 125\end{array}$

Kreatinkinase (U/1)

II $\quad(\mathrm{N}=29)$

III $(N=66)$

$15,0 \quad 17,0$

$\begin{array}{lllllll}224 & 276 & 426 & 594 & 683 & 828 & 891 \\ 177 & 192 & 275 & 435 & 502 & 539 & 564 \\ 152 & 164 & 216 & 283 & 289 & 307 & 333\end{array}$

Alkalische Phosphatase (U/I)

\begin{tabular}{rcccc} 
I & $(\mathbf{N}=71)$ & 145 & 150 & 156 \\
II & $(\mathbf{N}=42)$ & 127 & 133 & 174 \\
III & $(\mathbf{N}=66)$ & 148 & 215 & 228 \\
IV & $(\mathbf{N}=91)$ & 110 & 159 & 212 \\
V & $(\mathbf{N}=90)$ & 80,0 & 90,8 & 135 \\
\hline
\end{tabular}

$\begin{array}{rrrrrr}24,5 & 47,0 & 82,5 & 134 & 134 & 155 \\ 22,5 & 38,0 & 63,0 & 86,0 & 89,5 & 104 \\ & & & & & \\ 174 & 231 & 328 & 340 & 377 & 489 \\ 220 & 364 & 501 & 563 & 584 & 605 \\ 273 & 408 & 579 & 649 & 722 & 812 \\ 241 & 336 & 503 & 584 & 622 & 829 \\ 214 & 338 & 499 & 540 & 586 & 682\end{array}$

die Percentilwerte als auch die Mittelwerte mit den 95\%-Vertrauensbereichen als auch die Histogramme berücksichtigt. Hierbei ergab sich vorwiegend eine bessere Ubereinstimmung der Percentilwerte mit den Richtwertbereichen, die von der Arbeitsgruppe 1976 für viele Kenngrößen rein empirisch aufgestellt wurden. Diese ,empirischen Richtwerte' erwiesen sich in den letzten Jahren als erstaunlich zuverlässig zur Beurteilung von Patientenwerten.

Der Aufstellung von ,Richtwerten“ aus den gemessenen Referenzwerten lag immer das B̈estreben zu Grunde, möglichst praktikable Werte für die tägliche Routine zur Verfugung zu stellen. So ergaben sich z.B. für einige Kenngrößen in den verschiedenen Altersstufen nur minimale Unterschiede. In solchen Fällen wurden die Altersstufen zusammengezogen. Für einige Kenngrößen ist es nicht sinnvoll, Bereiche anzugeben. So wurden z.B. für Enżyme im Serum nur obere Richtwerte angegeben. Bei einigen Kenngrößen wurden zu enge Bereiche der Referenzwerte entsprechend der Definition des Richtwertbereichs sinnvoll erweitert.

Tabelle 6 zeigt die bearbeiteten Werte, die wir für die tägliche Beurteilung von Patientenwerten für praktikabel und sinnvoll halten.

Für die Kenngrößen Gesamt-Eiweiß und Kreatinin entstand die Frage, ob innerhalb der Altersstufe III (Kinder nàch dem 12. Monat) mit zunehmendem Alter die Werte ansteigen. Aus diesem Grund wurden die entsprechenden Altersverteilungen dargestellt (Abb. 1 und 2) Aus ihnen geht deutlich hervor, daß eine homogene Verteilung des individuellen Alters innerhalb der Altersgruppe vorliegt und keine steigende Tendenz zu beobachten ist. Man muß annehmen, daß sich der Übergang zu den etwas höher liegenden Erwachsenen-Werten erst jenseits des 16 . Lebensjahres abspielt.

Die katalytische Konzentration der alkalischen Phosphatase ist jenseits des 10. Lebenstages deutlich höher als vorher. In der Altersgruppe II (2.-12. Monat) liegt sie gegenüber den anderen Altersgruppen noch einmal etwas höher. Bei der großen Schwankungsbreite der Werte ist das aber nur unerheblich. Das kommt auch in dem Diagramm der Altersverteilung der Werte zum Ausdruck (Abb. 3). Die Wachstumsschübe mit besonders hohen Werten liegen individuell offensichtlich zu ganz unterschiedlichen Zeiten.

Bei der Kreatinkinase wurden innerhalb der Neugeborenen-Gruppe zahlreiche extrem hohe Werte gefunden. Vermutlich sind sie durch Geburtsbedingungen verursacht. Die Darstellung der Altersverteilung innerhalb der Neugeborenen-Gruppe (Abb. 4) zeigt, daß die Extremwerte nur innerhalb der ersten 10 Lebenstage auftreten. Da die Anzahl der Werte für die Zeit zwischen dem 10. und dem 30. Lebenstag sehr gering ist, werden für die Neugeborenen-Gruppe keine Richtwerte angegeben. 
Tab. 6. Zusammenstellung der Richtwerte bzw. Richtwertbereiche, die nach den Referenzwerten festgelegt wurden. Altersgruppen siehe Tabelle 2.

\begin{tabular}{|c|c|c|c|c|}
\hline Kenngröße & SI-Ei & heit & $\begin{array}{l}\text { Konv } \\
\text { Einhe }\end{array}$ & $\begin{array}{l}\text { entionelle } \\
\text { it }\end{array}$ \\
\hline Harnstoff & $\left.\begin{array}{r}\text { II } \\
\text { III }\end{array}\right\}$ & bis $7,5 \mathrm{mmol} / 1$ & $\left.\begin{array}{r}\text { I } \\
\text { III }\end{array}\right\}$ & bis $45 \mathrm{mg} / \mathrm{dl}$ \\
\hline Kreatinin & $\left.\begin{array}{c}\text { I } \\
\text { III }\end{array}\right\}$ & $\begin{array}{l}\text { bis } 106 \mu \mathrm{mol} / 1 \\
\text { bis } 88 \mu \mathrm{mol} / 1\end{array}$ & $\underset{\text { III }}{\text { I }}\}$ & $\begin{array}{l}\text { bis } 1,2 \mathrm{mg} / \mathrm{dl} \\
\text { bis } 1,0 \mathrm{mg} / \mathrm{dl}\end{array}$ \\
\hline Harnsäure & $\left.\begin{array}{r}\text { II } \\
\text { III }\end{array}\right\}$ & $120-350 \mu \mathrm{mol} / 1$ & $\left.\begin{array}{r}\text { I } \\
\text { III }\end{array}\right\}$ & $2,0-6,0 \mathrm{mg} / \mathrm{dl}$ \\
\hline $\begin{array}{l}\text { Gesamt- } \\
\text { Eiweiß }\end{array}$ & III & $\begin{array}{l}46-68 \mathrm{~g} / 1 \\
48-76 \mathrm{~g} / 1 \\
60-80 \mathrm{~g} / 1\end{array}$ & $\underset{\text { III }}{\text { II }}$ & $\begin{array}{l}4,6-6,8 \mathrm{~g} / \mathrm{dl} \\
4,8-7,6 \mathrm{~g} / \mathrm{dl} \\
6,0-8,0 \mathrm{~g} / \mathrm{dl}\end{array}$ \\
\hline Natrium & $\left.\begin{array}{r}\text { II } \\
\text { III }\end{array}\right\}$ & $130-145 \mathrm{mmol} / 1$ & $\underset{\text { III }}{\text { II }}\}$ & $130-145 \mathrm{mval}$ \\
\hline Kalium & $\underset{\text { III }}{\text { III }^{\prime}}$ & $\begin{array}{l}3,6-6,0 \mathrm{mmol} / 1 \\
3,7-5,7 \mathrm{mmol} / 1 \\
3,2-5,4 \mathrm{mmol} / 1\end{array}$ & III & $\begin{array}{l}3,6-6,0 \mathrm{mval} / 1 \\
3,7-5,7 \mathrm{mval} / 1 \\
3,2-5,4 \mathrm{mval} / 1\end{array}$ \\
\hline Calcium & $\left.\begin{array}{c}\text { II } \\
\text { III }\end{array}\right\}$ & $\begin{array}{l}1,75-2,70 \mathrm{mmol} / 1 \\
2,05-2,70 \mathrm{mmol} / 1\end{array}$ & $\underset{\text { IIII }}{\text { I }}\}$ & $\begin{array}{l}3,5-5,4 \mathrm{mval} / 1 \\
4,1-5,4 \mathrm{mval} / 1\end{array}$ \\
\hline Chlorid & $\left.\begin{array}{r}\text { I } \\
\text { III }\end{array}\right\}$ & $95-112 \mathrm{mmol} / 1$ & $\left.\begin{array}{r}\text { II } \\
\text { III }\end{array}\right\}$ & $95-112 \mathrm{mval} / 1$ \\
\hline $\begin{array}{l}\text { Anorgan. } \\
\text { Phosphor }\end{array}$ & II & $\begin{array}{l}1,6-3,1 \mathrm{mmol} / 1 \\
1,6-2,6 \mathrm{mmol} / 1 \\
1,1-2,0 \mathrm{mmol} / 1\end{array}$ & III & $\begin{array}{l}4,8-9,5 \mathrm{mg} / \mathrm{dl} \\
4,9-7,9 \mathrm{mg} / \mathrm{dl} \\
3,4-6,2 \mathrm{mg} / \mathrm{dl}\end{array}$ \\
\hline Eisen & $\left.\begin{array}{r}\text { I } \\
\text { II }\end{array}\right\}$ & $7,2-29 \mu \mathrm{mol} / \mathrm{l}$ & $\left.\begin{array}{r}\text { I } \\
\text { III }\end{array}\right\}$ & $40-160 \mu \mathrm{g} / \mathrm{dl}$ \\
\hline Bilirubin & $\left.\begin{array}{r}\text { II } \\
\text { III }\end{array}\right\}$ & bis $17 \mu \mathrm{mol} / 1$ & $\begin{array}{r}\text { III } \\
\text { III }\end{array}$ & bis $1,0 \mathrm{mg} / \mathrm{dl}$ \\
\hline Cholesterin & $\begin{array}{c}\text { I } \\
\text { II } \\
\text { III }\end{array}$ & $\begin{array}{l}\text { bis } 4,4 \mathrm{mmol} / 1 \\
\text { bis } 4,9 \mathrm{mmol} / 1 \\
\text { bis } 5,4 \mathrm{mmol} / 1\end{array}$ & $\begin{array}{l}\text { I } \\
\text { III } \\
\text { III }\end{array}$ & $\begin{array}{l}\text { bis } 170 \mathrm{mg} / \mathrm{dl} \\
\text { bis } 190 \mathrm{mg} / \mathrm{dl} \\
\text { bis } 210 \mathrm{mg} / \mathrm{dl}\end{array}$ \\
\hline $\begin{array}{l}\text { Glutamat- } \\
\text { Oxalacetat- } \\
\text { Transaminase }\end{array}$ & $\begin{array}{r}\text { I } \\
\text { III }\end{array}$ & $\begin{array}{l}\text { bis } 39 \mathrm{U} / 1 \\
\text { bis } 27 \mathrm{U} / 1 \\
\text { bis } 22 \mathrm{U} / 1\end{array}$ & $\begin{array}{r}\text { I } \\
\text { III }\end{array}$ & $\begin{array}{l}\text { bis } 39 \mathrm{U} / 1 \\
\text { bis } 27 \mathrm{U} / 1 \\
\text { bis } 22 \mathrm{U} / 1\end{array}$ \\
\hline $\begin{array}{l}\text { Glutamat- } \\
\text { Pyruvat- } \\
\text { Transaminase }\end{array}$ & $\left.\begin{array}{c}\text { II } \\
\text { III }\end{array}\right\}$ & $\begin{array}{l}\text { bis } 30 \mathrm{U} / 1 \\
\text { bis } 24 \mathrm{U} / 1\end{array}$ & $\underset{\text { III }}{\text { II }}\}$ & $\begin{array}{l}\text { bis } 30 \mathrm{U} / 1 \\
\text { bis } 24 \mathrm{U} / 1\end{array}$ \\
\hline $\begin{array}{l}\gamma \text {-Glutamyl- } \\
\text { Transpeptidase }\end{array}$ & $\begin{array}{r}\text { I } \\
\text { III }\end{array}$ & $\begin{array}{l}\text { bis } 150 \mathrm{U} / 1 \\
\text { bis } 100 \mathrm{U} / 1 \\
\text { bis } 20 \mathrm{U} / 1\end{array}$ & $\underset{\text { III }}{\text { II }}$ & $\begin{array}{l}\text { bis } 150 \mathrm{U} / 1 \\
\text { bis } 100 \mathrm{U} / 1 \\
\text { bis } 20 \mathrm{U} / 1\end{array}$ \\
\hline $\begin{array}{l}\text { Lactat- } \\
\text { Dehydro- } \\
\text { genase }\end{array}$ & II & $\begin{array}{l}\text { bis } 800 \mathrm{U} / 1 \\
\text { bis } 500 \mathrm{U} / 1 \\
\text { bis } 300 \mathrm{U} / 1\end{array}$ & III & $\begin{array}{l}\text { bis } 800 \mathrm{U} / 1 \\
\text { bis } 500 \mathrm{U} / 1 \\
\text { bis } 300 \mathrm{U} / 1\end{array}$ \\
\hline Kreatinkinase & $\begin{array}{r}\text { III } \\
\text { III }\end{array}$ & bis $85 \mathrm{U} / 1$ & $\underset{\text { III }}{\text { II }}\}$ & bis $85 \mathrm{U} / \mathrm{l}$ \\
\hline $\begin{array}{l}\text { Alkalische } \\
\text { Phosphatase }\end{array}$ & $\left.\begin{array}{c}\text { I } \\
\text { III } \\
\text { IV } \\
\mathbf{V}\end{array}\right\}$ & $\begin{array}{l}150-380 \mathrm{U} / 1 \\
130-700 \mathrm{U} / 1 \\
100-600 \mathrm{U} / 1\end{array}$ & $\left.\begin{array}{r}\text { I } \\
\text { IIII } \\
\text { IV } \\
\text { V }\end{array}\right\}$ & $\begin{array}{l}150-380 \mathrm{U} / 1 \\
130-700 \mathrm{U} / 1 \\
100-600 \mathrm{U} / 1\end{array}$ \\
\hline
\end{tabular}

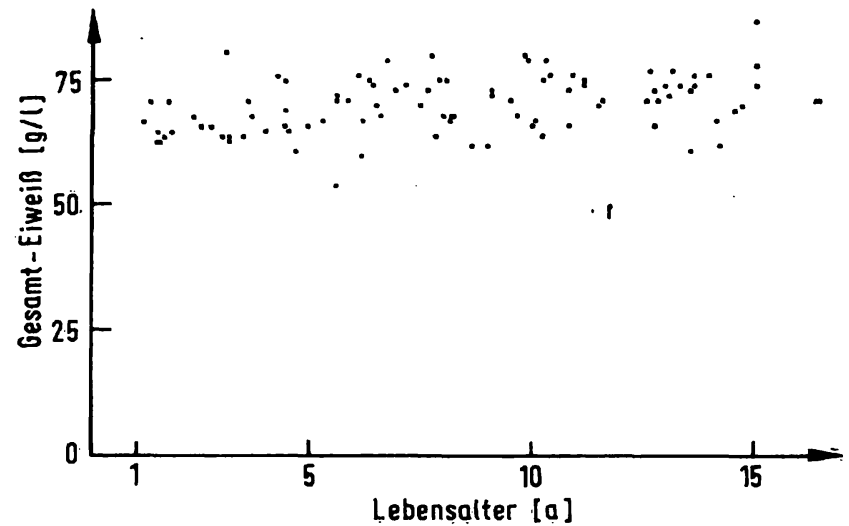

Abb. 1. Altersabhängige Verteilung der Wèrte für die GesamtEiweiß-Konzèentration innerhalb der Altersgruppe III (Kinder nach dem 12. Monat).

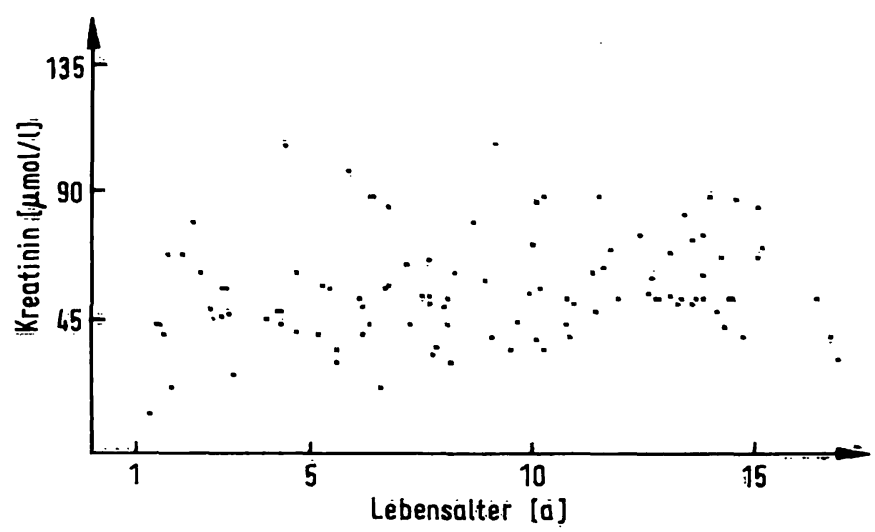

Abb. 2. Altersabhängige Veerteilung der Werte für die KreatininKonzentration innerhalb der Altersgruppe İII (Kinder nach dem 12. Monat).

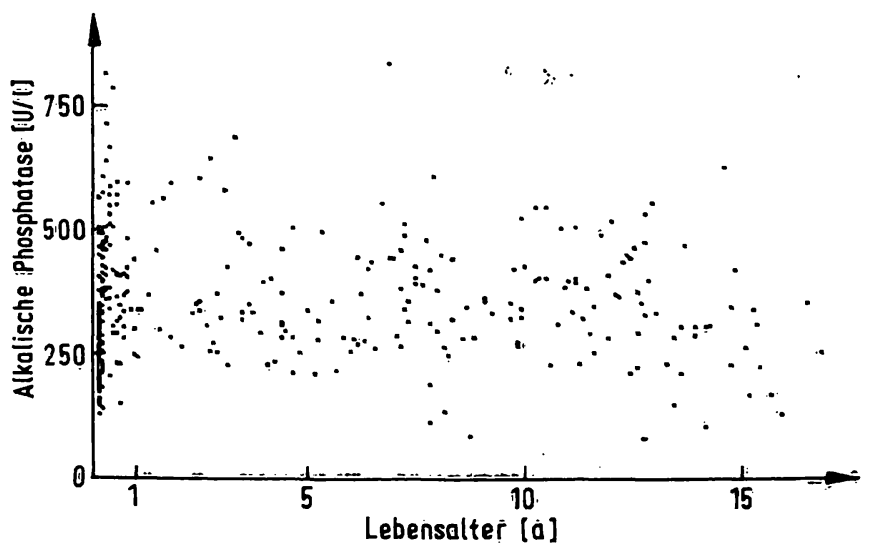

Abb. 3. Altersabhängige Verteilung der Werte fuì die kataly.tische Konzentration der Alkalischen Phosphatase innerhalb aller Aitersgruppen (Kinder vom 1. Lébeñstâg bis zum 16. Lebensjahr).

Für Eisen wurde ein breiter Referènzbereich gefunden. Dazu ist zu bemerken, daß die Eisenwerte der Kinger ohne Kontrolle des Blutbildes in die Studie aufgenommen wüden. Es kann nicht ausgeschlössen werden, daß die niedrigen Werte von Kindern mit einem klinisch noch nicht relevanten Eisenmangel stammen. Es erscheinit uns empfehlenswert, zur Beurteilung des Eisenhaushaltes der Kinder zusätzlich Ferritin zu bestimmen. 


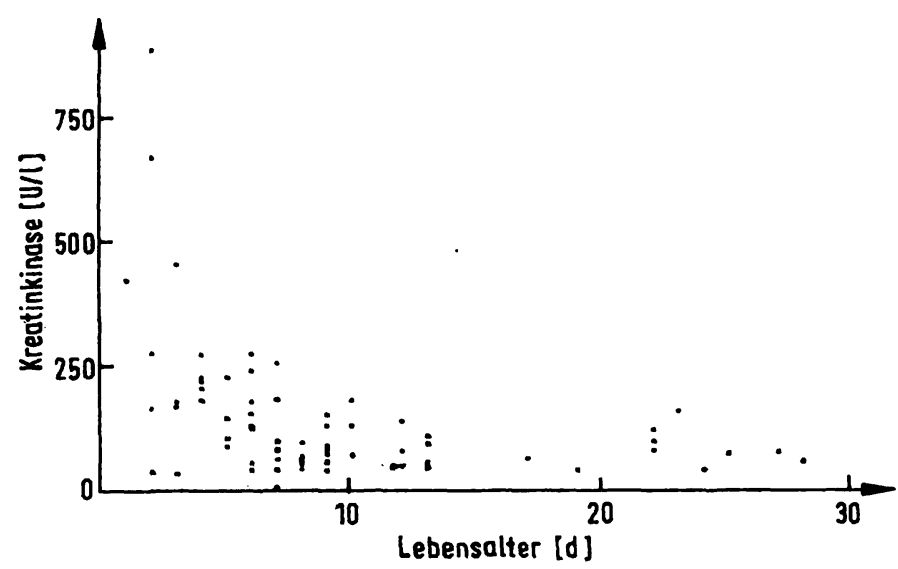

Abb. 4. Altersabhängige Verteilung der Werte für die katalytische Konzentration der Kreatinkinase innerhalb der Altersgruppe I (Neugeborene 1. Tag bis 4. Woche).

\section{Beurteilung der Studie}

Obwohl die Studie zu einem brauchbaren Ergebnis führte, gab es bei der Durchführung verschiedene Schwierigkeiten, so daß nicht alle geforderten Punkte erfüllt werden konnten.

Die Forderung, daß aus allen Kliniken die gleiche Anzahl von Werten pro Kenngröße und Altersstufe eingesandt wird, war nicht durchführbar, da es in einigen Kliniken nicht möglich war, genügend Neugeborene und Säuglinge, die die Minimalkriterien erfülten, in die Studie einzubeziehen. Zum Verständnis muß gesagt werden, daß nicht alle 19 Kenngrößen aus der Blutprobe eines einzelnen Kindes analysiert werden können. So waren pro Altersstufe wesentlich mehr als 5 Kinder zur Erstellung der Werte notwendig. Um genügend Werte pro Altersstufe zu erreichen, mußte also die Inhomogenität der eingesandten Werte in Kauf genommen werden. Die Anzahl der Werte prö Einsender liegen für die einzelnen Altersstufen und Kenngröße zwischen 5 und 20. Desgleichen konnten auch von verschiedenen Einsendem nicht alle Kenngrößen analysiẹt werden.

Die für einige Kenngrößen verwendeten unterischiedlichen Methoden erwiesen sich im Endeffekt in den vorliegenden Kombinationen nicht als gravierender Störfaktor. Bei der Analyse des gleichen Kontrollserums, das von einer zentralen Stelle an alle Teilnehmer verschickt wurde, zeigten sich signifikante Unterschiede zwischen verschiedenen Methoden für dieselbe Kenngröße. Diese Unterschiede waren jedoch wesentlich geringer als die biologische Varianz der Referenzwerte. Für die Brauchbarkeit der in der vorliegenden Studie ermittelten Richt- werte ist es sogar eher von Vorteil, wenn verschiedene Methoden angewandt wurden, da es nicht realistisch ist, anzunehmen, daß für alle gebräuchlichen Methoden gesonderte Richtwerte für das Kindesalter ermittelt werden können. Der Verzicht auf eine ausführliche Differenzierung der Methoden bei der Angabe von Richtwerten sollte von jedem Anwender als Aufforderung zu einer zumindest orientierenden Uberprüfung dieser Richtwerte unter den speziellen methodischen Gegebenheiten aufgefaßt werden.

Die Fehlerbreite der Analytik in den beteiligten Laboratorien erwies sich als sehr gering. In der 1 . Phase der Studie wurden die Proben jeweils an zwei aufeinander folgenden Tagen analysiert. Das bedeutete eine erhebliche Erschwerung der Studie. Die so ermittelten Werte zeigten aber so geringe Differenzen, daß in der 2. Phase der Studie auf diese Forderung verzichtet werden konnte.

Das wichtigste Ziel der Studie war die Aufstellung von Richtwerten, die für die tägliche Routine praktikabel und sinnvoll sind. Die interindividuellen Streuungen sind im Neugeborenen- und Säuglingsalter wesentlich größer als bei größeren Kindern und Erwachsenen. Das ist einer der Gründe, die uns veranlaßten, aus den gemessenen Referenzwerten Richtwerte abzuleiten. Ein weiterer Grund ist in dem Bestreben zu sehen, von der ,Zahlengläubigkeit' zu einer sinnvollen Einordnung des Laborwertes zu kommen. Unter dem Gesichtspunkt der Praktikabilität werden die Werte verschiedener Altersstufen zusammengefaßt, wenn sie sich nur geringfügig unterscheiden. Sie wurden in den Mittelwerten und Percentilen jedoch getrennt ausgedruckt, um die Gleichartigkeit verschiedener Altersstufen zu dokumentieren.

Eine interessante Beobachtung der Studie ist, daß die gemessenen Werte erstaunlich gut mit den von der Arbeitsgruppe 1976 rein empirisch festgelegten Richtwerten übereinstimmen. Meistens war die Ubereinstimmung größer mit den Percentilen als mit den Mittelwerten und dem $95 \%$-Vertraueñsbereich. Weiter zeigte sich eine gute Ubereinstimmung der gemessenen Werte für viele Kenngrößen mit einer in der Universitäts-Kinderklinik 1977 durchgeführten retrospektiven Studie anhand von Krankenblättern, wobei die einbezogenen Kinder ebenfalls die Minimalkriterien erfüllen mußten.

Die durchgeführte Studie ist ein brauchbares Modell für ähnliche gemeinsame Werteermittlungen. Die Ausdehnung auf weitere klinisch-chemische, hämatologische und hämostaseologische Kenngrößen im Kindesalter ist wünschenswert.

\section{Danksagung}

Wir danken Herm Dipl. math. R. Rossner vom Institut fuir Medizinische Dokumentation und Statistik, der uns einen Teil der benötigten Programme zur Verfügung gestellt hat, und dem Rechenzentrum dex Universität Freiburg, auf dessen Groß-

rechner UNIVAC 1100/80 die Auswertungen durchgeführt wurden, für die freundliche Unterstützung.

Frau Christa Schelzig danken wir für die sorgfailtige Mitarbeit bei der Datenverarbeitung. 


\section{Literatur}

1. Fawcett, J. K. \& Scott, J. E. (1960) J. Clin. Pathol. 13, $156-159$.

2. Paulson, G., Ray, R. \& Sternberg, J. (1971) Clin. Chem. 17 [Abstr. 63. Med. Papers] 644.

3. Seelig, H. P. \& Wüst, H. (1969) Ärztl. Lab. 15, 34-39.

4. Helger, R., Rindfrey, H. \& Hilgenfeldt, J. (1974) Z. Klin. Chem. Klin. Biochem. 12, 344-349.

5. Liappis, N. (1979) Klin. Pädiat. 191, 66-71.

6. Kortüm, M. \& Kling, O. (1972) Ärztl. Lab. 18, 33-36.

7. Kageyama, N. (1971) Clin. Chim. Acta 31, 421-426.

8. Weichselbaum, T. E. (1946) Amer. J. Clin. Pathol. 16 [Techn. Sect. Vol. 10] 40-49.

9. Zilversmit, D. B. \& Davis, A. K. (1950) J. Lab. Clin. Med. $35,155-160$.

10. Trinder, P. (1956) J. Clin. Pathol. 9, 170-172.

11. Lauber, K. (1965) Z. Klin. Chem. 3, 96-99.

12. Stoockey, L. L. (1970) Anal. Chem. 42, 779-781.

13. Jendrassik, L. \& Gróf, P. (1938) Biochem. Z. 297, $81-89$.

14. Wahlefeld, A.-W., Herz, G. \& Bernt, E. (1972) Scand. J. Clin. Lab. Invest. 29, Suppl. 126, Abstr. 11. 12.

15. Röschlauu, P., Bernt, E. \& Grúber, W. (1974) Z. Klin. Chem. Klin. Biochem. 12, 403-407.

16. Stähler, F., Gruber, W., Stinshoff, K. \& Röschlau, P. (1977) Med. Lab. 30, 29-37.

17. Empf. d. Dt. Gesellsch. f. Klin. Chemie (1972) Z. Klin. Chem. Klin. Biochem. 10, 182-192.

18. Szász, G., Weimann, G., Stähler, F., Wahlefeld, A.-W. \& Persijn, J.-P. (1974) Ż. Klin. Chem. Klin. Biochem. 12, [Abstr. 6.4 Biochem. Analytik '74] 228.

19. Recömm. German. Soc. Clin. Chem. (1977) Ẑ. Klin. Chem. Klin. Biochem. 15, 255-260.

20. Todorov, J. (1970) Klinische Laboratoriumsuntersuchungen im Kindesalter, VEB Verlag Volk und Gesuñdheit, $\mathrm{X}, 1114 \mathrm{~S}$. Berlin.

21. Sitzmann, F. C. (1976) Normạlwerte, H. Marseille Vèrlag, 208 S., München.

22. American Association for Clinical Chemistry, Pediatric Clinical Chemistry, Winton=Salem 1977.

Frau Professor Dr. Irene Witt

Klinisch-Chemisches und Biochemisches Labor Universitäts-Kinderklinik

Mathildenstraße 1

D-7800 Freiburg

Priv.-Doz. Dr. Chr. Trendelenburg

Klinisch-Chemisches Institut

Kàtharinenhospital

Kriegsbergstraße 60

D-7000 Stuttgart 1 